\title{
An Enhanced Service Flow Management Scheme for IEEE 802.16 Broadband Wireless Access Systems
}

\author{
Juma Modie Mwamafundo, Sang Kipkorir, and Felix Musau
}

\begin{abstract}
While the protocols have been proposed to support QoS (quality of service) guarantees for a variety of applications, the WiMax/IEEE 802.16 standard does not specify a mandatory scheduling architecture to fulfill stringent QoS requirement for the different classes of traffic. Only the framework is established in the standard which provides an opportunity for developers to design efficient scheduling mechanism to fulfill stringent $\mathrm{QoS}$ requirement for the different classes of traffic. The scheduling scheme requires catering for rigorous QoS guarantee for all types of service flows as specified in the standard, enabling fairness and facilitating the traffic of lower priority. In this paper, we propose a Service Flow Management Scheme for the IEEE 802.16 Broadband Wireless Access systems that can provide QOS support that enhances performance through a scheduler that performs optimally and efficiently.
\end{abstract}

Index Terms-BWA, IEEE 802.16, QoS, scheduling, WiMAX.

\section{INTRODUCTION}

At present, BWA (Broadband Wireless Access) networks have proved to be a viable solution providing service to personal and commercial clients especially those with demands for high speed Internet access.

The Worldwide Interoperability for Microwave Access referred as (WiMax) system is based on the IEEE 802.16-2004 standard [1] and its amendment IEEE 802.16e [2]. The IEEE 802.16 standard defines the physical (PHY) and medium access control (MAC) layer of fixed and mobile broadband wireless access system. IEEE 802.16 standard is intended to meet and better the expectation of broadband cabled networks especially in areas considered difficult to deploy such networks. This is achieved by providing a wireless communication on a broadband scale, which forms a striking option to broadband wired networks such as fibre optic links or copper wires.

Deployment of BWA systems does not necessitate cabling to connect the stations which make it easy for change and

Manuscript received May 22, 2011; revised July 3, 2012. This work was financially supported by the School of Information Science and Engineering Central South University, Changsha, Hunan Province, 410083, P.R China.

J. M. Mwamafundo was with the School of Information Science and Engineering Central South University, Changsha, Hunan Province, 410083, P.R China. He is now with ministry of Nairobi Metropolitan Development as Chief ICT Officer, Nairobi, Kenya (e-mail: j.modie@ nairobimetro.go.ke).

S. Kipkorir was with the School of Information Science and Engineering Central South University, Changsha, Hunan Province, 410083, P.R China. He is now with the Jomo Kenyatta University of Science and Technology, Nairobi, Kenya (e-mail: kipkorirsang@gmail.com).

F. Musau is with the School of Information Science and Engineering Central South University, Changsha, Hunan Province, 410083, P.R China (e-mail: musaunf@gmail.com). future expansion, hence making it cost effective and allowing extensive mobility. In addition, WiMAX has the power to cover, in Line of Sight (LOS), a range of $50 \mathrm{~km}$ in point to point transmissions with a throughput of about $72 \mathrm{Mbps}$ and in Non-Line of-Sight (NLOS) a range of $6.5 \mathrm{~km} \mathrm{[3].}$

The rest of the paper is organized as follows. Section II, explains the IEEE 802.16 standard in brief. Section III is related work from some of the relevant proposals reported in the literature. In Section IV, we introduce the details of the proposed architecture. Section V is the conclusion and future work.

\section{THE IEEE 802.16 STANDARD}

The standard defines two operation modes: Mesh mode and Point-to-Multipoint (PMP) mode [4]. Mesh mode supports direct communications between Subscriber Stations SSs without the need for a Base Station BS. For PMP topology, a controlling BS connects multiple SSs to various public networks as shown in the Fig. 1.

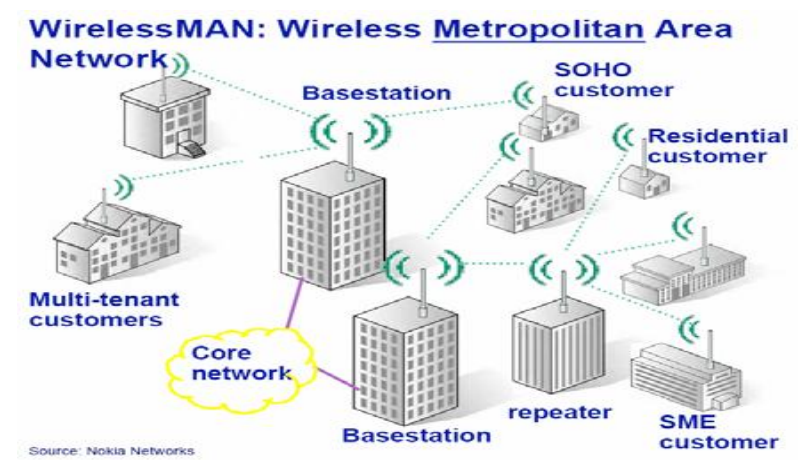

Fig. 1. Point-to-multipoint wireless metropolitan area network.

In this work, we consider the latter where the standard defines an air interface for fixed Point-to-Multipoint BWA that is competent of providing various services. The BS schedules the traffic flow, communication between BS and SSs are bidirectional, downlink channel (BS to SS) is in broadcast mode and uplink channel (SS to BS) is shared by various SSs. The standard supports two types of duplex mode, Time Division Duplex (TDD) and Frequency Division Duplex (FDD).The TDD frame comprises of downlink and uplink subframes, the duration and the number of subframe slots are defined by the BS scheduler. The downlink subframe has downlink map (DL map) containing information about the duration of subframes and which time slot belongs to a particular SS as the downlink channel and uplink map (UL map) consists of information element (IE) which includes transmission opportunities [5]. The WiMAX MAC layer caters for an interface between the higher 
transport layers and the physical layer. It picks out MAC service data units (MSDUs) packets from the upper layer and forms them into MAC protocol data units (MPDUs) for sent transmission and likewise for received transmission. Its design encompasses a convergence sublayer (CS) that can interface with a range of higher-layer protocols, such as ATM, TDM, Ethernet, IP, and any future protocol. Apart from providing mapping to and from higher layers, the CS supports MSDU header suppression to reduce the higher layer overheads. [6] The transmission is structured into frames, and every frame is split into three phases: the broadcast phase where the BS uses it to broadcast the structure of the frame, the downlink phase that is for transmitting data in the downlink direction, and the uplink phase which is further divided into a contention sub-phase for conveying control information from the SS to the BS and a data sub-phase used to transmit data in the uplink direction. Apparently, the grants in each frame depend on the scheduler algorithm implemented by the BS and on the resources requested by each connection. The status of the resource requests in the downlink is all available to the BS: it only checks the size of the connection queues. As for the uplink, the BS does not have full access to the connection uplink queues. Signalling mechanism must take place to convey this valuable information to the BS. The standard offers four bandwidth request mechanisms: a contract based mechanism, a mechanism based on a polling mechanism, a piggyback scheme and a contention process mechanism. A connection will use one or another request mechanism depending on the type connection [7]. Supporting QoS is an essential part of the MAC layer design in WiMAX. It uses QoS design standard similar to the Data Over Cable Service Interface Specification (DOCSIS) cable modem standard. In a MAC connection oriented architecture, data between BSs and SSs is transmitted in the context of connection. Each connection is identified in the (MPDU) by a connection identifier (CID) which provides mapping to a service flow identifier (SFID).SFID is an important concept in the MAC layer, it provides a mapping, to the QoS parameters for a particular data entity.

[1] WiMAX defines the following QoS classes that should be supported by the BS in order to support a wide variety of applications: UGS (Unsolicited Grant Service) supports constant bit-rate (CBR) connections, e.g. VoIP, rtPS (Real-Time Polling Service) characterized by a variable bit-rate, and by their time constraint requirements, i.e., limited maximum delay and limited jitter, e.g. video-conference, nrtPS (Non-Real-Time Polling Service) used by non-real-time applications requiring better than best effort services, e.g., intensive file transfer, data base applications, etc. These types of applications are not sensible to delay, and BE (Best Effort) such as HTTP, service has the lowest priority. The standard proposes the mapping between the bandwidth request mechanisms and the connection type. However, the numerical values of the various parameters to be used by each mechanism are unspecified, e.g. in UGS connections, the amount of reserved bandwidth. As for non-UGS flows, the standard leaves the scheduling algorithm undefined [7].

\section{RELATED WORK}

Many works have proposed new packet scheduler architecture for 802.16 networks, in order to provide different levels of QoS guarantees for various applications. This is as a result of lack of standardization for the Admission Control and Uplink Scheduling algorithm for BE, rtPS and nrtPS service flows in the 802.16 standard. [2] Architecture is proposed which introduces a framework for the scheduling algorithm and admission control policy for 802.16. Also system parameters are suggested that may be used, and identify traffic characteristics for which the network can provide QoS. [3], [4] Give a precise description of the proposed architecture and more background on the 802.16 standard. Authors in [5] propose a scheduler in which the priority is based on the channel and service quality. Work in [6] elaborates a scheduler for the uplink that uses hard QoS and best effort for the BS, ensuring fairness. [7] Presents scheduling architecture based on weighted fair queueing WFQ. [8] Proposes a scheduling architecture for both 802.16 and data over cable service interface specifications (DOCSIS). [11] Focuses on the use of link adaptation for carrying out the scheduling function. An uplink scheduling architecture based on requests deadlines is proposed [12]. [13] Detailed a deficit fair priority queue scheduling algorithm, which is deployed to cater for the different types of traffic flows for both uplink and downlink traffic.

\section{PRoposed ARChiteCture}

In the IEEE 802.16 standards, some mechanisms to guarantee QoS have been defined. Our proposed architecture have taken these into consideration and introduced other modules to ensure connections realize maximum network usage. Fig. 2a and Fig. 2b below shows our proposed architecture.

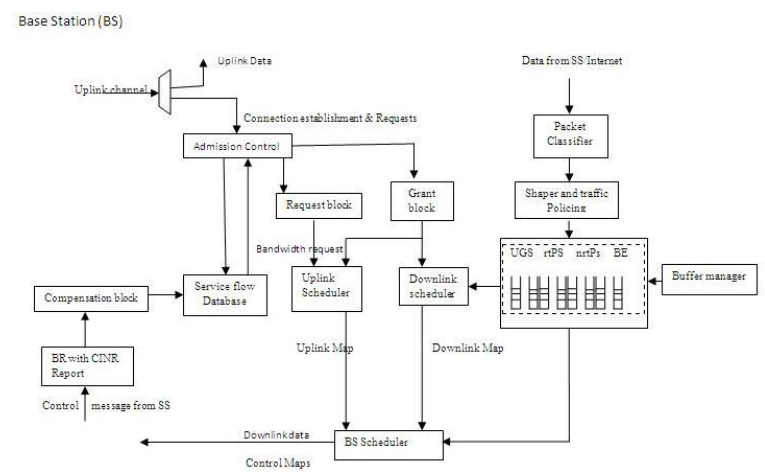

Fig. 2a. Proposed QoS architecture for IEEE 802.16 at the base station

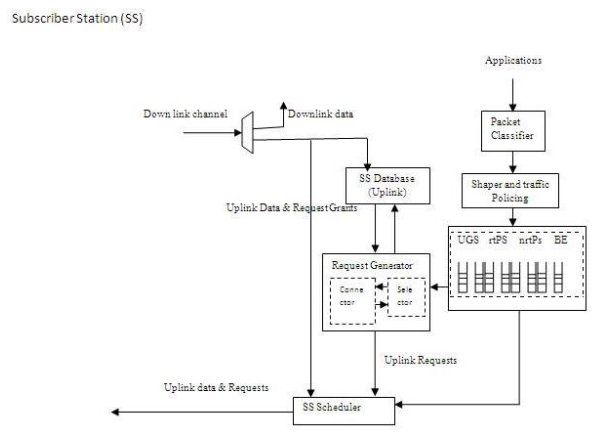

Fig. 2b. Proposed QoS architecture for IEEE 802.16 at the subscriber sation 
Let us now look at each module of this architecture.

\section{A. Admission Control}

This is where a new request for connection can be granted or not depending on the remaining free bandwidth. It is a very crucial aspect in QoS architecture so as to avoid network overload or starvation of some flows, determined on the basis of both its traffic class or requirements and the available network resources. BS needs an admission control policy to determine whether QoS of a connection can be achieved. On our case, an admission control policy is implemented using the Minimum Reserved traffic rate where all the DSD /DSC/ DSA requests are collected, and update the estimated available bandwidth $\left(C_{a}\right)$ based on bandwidth variations [19].

$$
C_{a}=C_{\text {total }}-\sum_{i=0}^{I} \sum_{j=0}^{J_{i}-1} r_{\min }(i, j)
$$

where $r_{\min }(i, j)$ is the Minimum Reserved traffic rate of the $j$ th connection in the $i$ th class of service flow, $C_{\text {total }}$ is the total capacity of the wireless link. Connections, where $r_{\min }$ equals to zero, can constantly be accepted by our admission policy, otherwise, connections will not be granted. They mostly have the lowest priority. Their connections will be interrupted anytime until the QoS needs of all other connections are sufficiently meet. When a new service flow comes or an old service flow requests to change its QoS, the rule, $C_{a} \geq 0$ holds.

\section{B. Packet Classifier/ Shaper and Traffic Policing}

These modules determine the type of service to be assigned to an incoming traffic and, if need be, police it for complying with the specifications approved during the connection establishment phase. The parameters, Traffic Shaper and policing use, are lay down as soon as the Admission Control allows a new connection. The classification of the data packets is necessary for identifying the service associated with the connection. This identification is based on the IEEE 802.1p recommendation [11]. Packets received are classified by the packet classifier and buffered into one of the per class queues as rtPS, nrtPS and BE. UGS and ertPS service classes are not subject to scheduling, as they receive a constant reserved amount of bandwidth frame-by-frame. In the BS, the classifier timestamps every arriving packet according to its arrival time for each connection. This information is used by the Buffer manager to identify when the packet timeout expires, i.e., if the waiting time of such a packet in queue is over and above its maximum latency (deadline). At the SS, Traffic shaping and policing is necessary to guarantee conformance between actually generated traffic and negotiated traffic at connection setup.

\section{Downlink/Uplink Queues}

These realize the data queues for the downlink and uplink connections. A new queue is created in one of these modules, when the Admission Control accepts a new connection, depending on the way the communication flows. The queues are independent, so each could implement a different scheduling policy. The queues also could be grouped by service flows to solve scalability issue generally experienced in highly populated systems.

\section{Buffer Manager}

Buffer manager is used for queue length control and packet dropping when necessary. It is assumed that per-class queues are infinite in size. Hence, the buffer manager purges queues of all the packets buffered for a time longer than their maximum tolerated delay, at the beginning of each frame. The aim of purging is to do away with meaningless compensation over already terminated packets and bandwidth waste to transmit timely worthless packets. The same objective is achieved in periodic buffer cleaning as readjusting in [17], but with no need to tune the maximum leading/lagging amount per flow.

\section{E. Compensation Block}

The compensation block classifies a flow at the beginning of each frame, i.e. CID as perceived a "good" or a "bad" channel depending on the CINR reports received by the intended target SSs. Hence, CIDs meant for a SS, sensing a lossy channel are marked as banned.

\section{F. Bandwidth Requests with CINR Reports}

In this case, each SS monitors the state of its channel constantly, predicting future channel state, and sends this information to the scheduler in the BS. The Compensation block is the component that ensures the BS is aware of the channel state. Gathering information on the channel quality that the BS receives through the bandwidth request (BR) messages sent by SSs, messages normally used by SSs for bandwidth requests, comprise a carrier to interference and noise ratio, CINR, report [1].

\section{G. Service Flow Database/ SS Database}

Information of all active connections is maintained in these modules. The QoS parameter change associated with the connections are updated into these modules. A new record is set up when a new connection is established by the Admission Control. Information in these modules is used by the schedulers to decide the QoS requirements of each active connection.

\section{H. Request Block}

Request block in the BS handles the incoming request. This is where the flow type and/or identifier of the connection from the request is determined. Once the flow type or identifier is acknowledged, the request is queued and set waiting to be served.

\section{BS Scheduler}

Traffic scheduling offers the desired QoS guarantee to the traffic crossing the air link, while optimally utilizing link resources. The BS Scheduler determines the structure of each frame by obtaining information from the Grant block, Downlink Queues, Request block and Service flow Database where the Uplink Scheduler is used to schedule the uplink resources between the connections with pending request. The Uplink Scheduler prepares a broadcast message, Uplink-Map, having the identifiers of all the connections granted to transmit in the current frame. Hence, the Downlink Scheduler computes the grants allocated in the downlink direction 
according to Downlink Queues state and the grants produced in downlink.

\section{J. SS Scheduler}

The UL-MAP message of the broadcast is examined by the SS Scheduler to identify the grants allocated in the current frame to the SS's then decides depending on the resources to be shared among the active connections of the SS.

\section{K. Grant Block}

This block produces the periodical grants. How often and amount of the grants generated are established during the initial set up process, therefore, the Admission Control module must update this module after accepting a new connection. The BS Scheduler need to be updated on the grants generated inorder to be considered by the allocation process.

\section{Request Generator}

The IEEE 802.16 standards is so exact in request and grant mechanism yet no stipulated design is provided. Hence, we propose an efficient technique for it. While the BS polls each SS at certain intervals, SS can solely generate and send bandwidth requests. Bandwidth requests may be aggregate or incremental. If BS receives an incremental bandwidth request, it shall add the quantity of bandwidth requested to its current perception of the bandwidth requirements of the connection. Whereas the BS receives an aggregate bandwidth request, it shall replace its perception of the bandwidth requirements of the connection with the quantity of bandwidth requested [1]. As depicted in Fig. 2, the Connector sub module in SS is accountable for generating one request for each connection in each frame; this is achieved as per the amount of data in their queues. The self-correcting nature of the Request and Grant protocol ensures that SSs periodically use aggregate bandwidth Requests. The period may be a function of the QoS of a service and of the link quality. Unless there is an aggregate request to send, in all cases, connections would generate incremental request as shown [20].

$$
\begin{gathered}
I n C_{i}=\sum_{i=\text { Lastaccepted }}^{\text {cuspert }} g_{i}+\left(q_{i} \text { len }\right)_{\text {curvent }}-\left(q_{i} \text { len }\right)_{\text {LastAciepted }} \\
A g g_{i}=(\text { qi.len })_{\text {current }}
\end{gathered}
$$

where $A g g_{i}$ and $I n c_{i}$ are the aggregate and incremental requests for connection $i$ respectively, $\left(q_{i} \text {.len }\right)_{\text {current }}$ is the current queue length of the $i$ th connection and $\left(q_{i} . l e n\right)_{\text {LastAccepted }}$ is the length of that queue when the last request of that connection was established by Request Selector. $\sum_{i=\text { LastAccepted }}^{\text {current }} g_{i} j$ is the total grant allocated to connection $i$ from the time the last request of the connection was established upto the current time. The Selector in each SS examines the requests generated in Connector, and from their types and also their connection QoS attributes picks, and sends at most one of them. The main concept applied is as follows. First each queue is allocated a weight according to its QoS parameters, and then in each round the selector selects one request by multiplying the weight and length of the queues and choosing the greatest.

\section{CONCLUSION}

In this paper, we have proposed architecture to provide QoS flow management scheme over IEEE 802.16 BWA systems that enhances performance for the different service flows as specified in the standard, applying a compensation technique for channel errors that is, in most cases, generalized to other schedulers.

In future, we plan to conduct extensive simulations to ascertain its practical implementation to determine the extent of performance and stability of this QoS architecture. Additionally, comparisons and analysis of the different implementation advancements for each module in the architecture, mainly scheduling algorithms also need to be done.

\section{REFERENCE}

[1] Local and Metropolitan Area Networks Part 16: Air Interface for Fixed Broadband Wireless Access Systems, IEEE 802.16 Standard, 1 October 2004,

[2] Air Interface for Fixed and Mobile Broadband Wireless Access Systems, IEEE802.16e, part 16, Feb 2006.

[3] G. S. Paschos, I. Papapanagiotou, C. G. Argyropoulos, and S. A. Kotsopoulos, A Heuristic Strategy for IEEE 802.16 WiMAX scheduler for Quality of Service

[4] H. Safa, H. Artail, M. Karam, R. Soudah, and S. Khayat, New Scheduling Architecture for IEEE 802.16 Wireless Metropolitan Area Network.

[5] European Telecommunications Standards Institute, Broadband Radio Access Networks (BRAN), HiperMAN Physical (PHY) layer, ETSI TS102 177 V1.2.1, 2005-01, 2005.

[6] Air Interface for Fixed Broadband Wireless Access System, Amendment 2: Physical and Medium Access Control Layers for Combined Fixed and Mobile Operation in Licensed Bands and Corrigendum 1, IEEE 802.16e, IEEE Standard for local and metropolitan area networks, Feb 2006.

[7] J. Delicado, L. Orozco-Barbosa, F. Delicado, and P. Cuenca, "A QoS-aware protocol architecture for WiMAX," Electrical and Computer Engineering, CCECE '06, 2006, pp. 1779-1782

[8] K. Wongthavarawat and A. Ganz, Packet scheduling for QoS support in IEEE 802.16 broadband wireless access systems, Wiley, c2003.

[9] G.-S. Chu, D. Wang, and S. Mei, "A QoS Architecture for the MAC Protocol of IEEE 802.16 BWA System," Presented at IEEE International Conference on Communications, Circuits and Systems and West Sino Expositions, June 2002.

[10] S. Maheshwari, "An Efficient QoS Scheduling Architecture for IEEE 802.16 Wireless MANs," Master's thesis, Indian Institute of Technology, Bombay, 2005.

[11] Q. Liu, X. Wang, and G. B. Giannakis, "Cross-Layer Scheduler Design with QoS Support forWireless Access Networks," Second International Conference on Quality of Service in Heterogeneous Wired/Wireless Networks, Aug. 22-24, 2005.

[12] N. Liu, X. Li, C. Pei, and B. Yang, "Delay Character of a Novel Architecture for IEEE 802.16 Systems," in Proceedings of the Sixth International Conference on Parallel and Distributed Computing, Applications and Technologies PDCAT.05, 2005.

[13] M. Hawa and D. W. Petr, "Quality of Service Scheduling in Cable and Broadband Wireless Access Systems," Tenth International Workshop on Quality of Service, pp. 247- 255, May 2002.

[14] S. Ramachandran, "Link Adaptation Algorithm and Metric for State University," [Online]. Available: http://scholar.lib.vt.edu/theses/available/etd02272004133201/unrestri cted/ramachandran_thesis.pdf, February 12, 2004

[15] J. Freitag and N. L. S. da Fonseca, "Uplink Scheduling with Quality of Service in IEEE 802.16 Networks," Institute of Computing, State University of Campinas 13089- 971, S ao Paulo, Brazil. 2007.

[16] J. Chen, W. Jiao, and Q. Guo, "An Integrated QoS Control Architecture for IEEE 802.16 Broadband Wireless Access Systems," Lucent Technologies, Bell Labs Research China, 2005. 
[17] Information Technology - Telecommunications and Information Exchange between Systems - Local and Metropolitan Area Networks Common Specifications - Part 3: Media Access Control (MAC) Bridges (ANSI/ IEEE Std 802.1D), ISO/IEC (ANSI/IEEE) Std. 15 802-3, 1998.

[18] Y. Yi et al., "W2F2Q: Packet Fair Queuing in Wireless Packet Networks," Wksp. Wireless Mobile Multimedia, Aug. 2000.

[19] J. Chen, W. Jiao, and H. Wang, "A Service Flow Management Strategy for IEEE 802.16 Broadband Wireless Access Systems in TDD Mode," in Proc. IEEE International Conf. on Communications, ICC 2005, May 2005, pp. $3422-3426$.

[20] H. S. Alavi, M. Mojdeh, and N. Yazdani, "A Quality of Service Architecture for IEEE 802.16 Standards," Dept. of ECE University of Tehran, Tehran, Iran 2005 Asia-Pacific Conference on Communications, Perth, Western Australia, 3 - 5 October 2005.

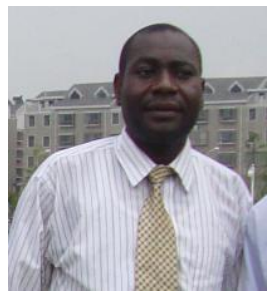

Juma Modie Mwamafundo born in Nairobi, Kenya on $22^{\text {nd }}$ February, 1977. Studied Bsc Computer Science at Islamic University in Uganda, Mbale, Uganda, 2002 and Msc Computer Science and Technology at Central South University, Changsha, Hunan, P.R. China, 2011. He is currently the Chief ICT Officer in the Ministry of Nairobi Metropolitan Development under e-government Nairobi, Kenya.

He is interested in mobile and wireless computer network technologies.

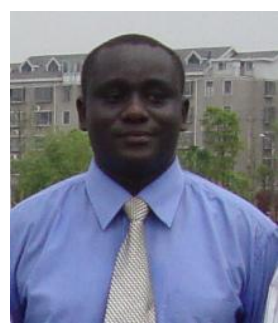

Sang Kipkori completed Msc Computer Science and Technology at Central South University, Changsha, Hunan, P.R. China, 2011. He is currently at Jomo Kenyatta University of Science and Technology, Nairobi, Kenya. He researched and is interested in mobile handover technologies in wireless networks.

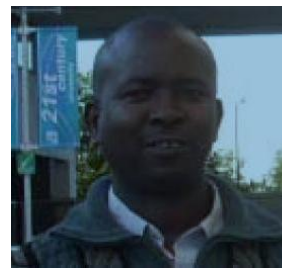

Felix Musau Kenyan born in 1970. Studied MEng (Computer Science Technology and Applications), 2003. P. G. D. Comp Sc, BED (Sc) (hons), 2001 and now pursuing PhD in Computer Science and Technology at Central South University, Changsha, Hunan, P.R. China. 2003-2004 was teaching at Kenya Science Teachers College. 2004-2005-System Engineer ,Kenya Polytechnic, Nairobi 2007-2008 was chief examinations officer, Information and communication Technology department, Kenyatta University. Research area of interest are trust in peer to peer networks, mobile ad hoc networks, security of wireless sensor networks and semantic web technology, Digital Image Processing. 